Article

\title{
Bandwidth and Gain Enhancement of Endfire Radiating Open-Ended Waveguide Using Thin Surface Plasmon Structure
}

\author{
Abhishek Kandwal ${ }^{1}\left(\mathbb{D}\right.$, Zedong Nie ${ }^{1, *} \mathbb{D}$, Jingzhen Li $^{1}$, Yuhang Liu ${ }^{1}$, Louis WY. Liu ${ }^{2, *(D)}$ \\ and Ranjan Das ${ }^{3}$ (D) \\ 1 Shenzhen Institutes of Advanced Technology, Chinese Academy of Sciences, Shenzhen 518055, China; \\ abhishek@siat.ac.cn (A.K.); lijz@siat.ac.cn (J.L.); yh.liu2@siat.ac.cn (Y.L.) \\ 2 EEIT Department, Vietnamese German University, Binh Duong 820000, Vietnam \\ 3 Indian Institute of Technology, Bombay 400076, India; ranjandas@ee.iitb.ac.in \\ * Correspondence: zd.nie@siat.ac.cn (Z.N.); liu.waiyip@vgu.edu.vn (L.W.L.)
}

Received: 24 April 2019; Accepted: 2 May 2019; Published: 7 May 2019

\begin{abstract}
This paper proposes a technique to enhance the bandwidth and gain of an endfire radiating open-ended waveguide using a thin slow-wave surface plasmon structure. Mounted in the E-plane of the stated waveguide, a thin corrugated slow-wave structure has been used in conjunction with a waveguide transition to generate an endfire electromagnetic beam. An efficient mode conversion from waveguide transition to the corrugate plate resulted in the improved performance of the design. An impedance bandwidth from $8 \mathrm{GHz}$ to $18 \mathrm{GHz}$ has been achieved along with a gain enhancement from $7 \mathrm{dBi}$ to $14.8 \mathrm{dBi}$ using the proposed hybrid design. Endfire radiations have been obtained with a beam width of less than $25^{\circ}$ through the proposed hybrid design with an efficiency of about 96 percent.
\end{abstract}

Keywords: open-ended waveguide; slow-wave; endfire; radiation; dispersion; gain

\section{Introduction}

Rectangular waveguides have been in use for many decades for a range of communication applications. In principle, energy propagates along the waveguide in transverse electric modes (TE modes) with little energy loss. The lowest frequency in which a certain TE mode can propagate, which is known as the cutoff frequency of that mode, depends on the cross-sectional dimensions of the waveguide. The mode with the lowest cutoff frequency is known as the fundamental mode of the waveguide. Although other higher order modes do co-exist with the fundamental mode, the even order modes cannot propagate along the E-plane of the waveguide. Recently, the concept of spoof surface plasmon polaritons (SSPPs) has attracted a lot of interests from the microwave community, in part because of the following features and merits: groundlessness, high field confinement and feasibility of guiding an electromagnetic energy in surface modes. The effective exploitation of these features require a reliable evaluation of the important parameters of spoof surface plasmon (SSP)-based transmission lines (TLs). Surface plasmons (SPs) are highly localized electromagnetic (EM) waves with exponential field decay from the interface of metal and dielectric.

Recent applications of SSPs highlight the importance of a convenient and quick design procedure for the SSP structures. In a traveling-wave antenna, there are typically two modes of wave propagation: leaky modes and surface modes. A leaky mode propagates along the guiding body of the antenna with a group velocity greater than the speed of light, radiating electromagnetic energy in all directions other than the endfire direction. A surface mode propagates along the guiding body of the antenna with a group velocity less than the speed of light, with the electromagnetic field very confined on 
the surface or along an edge. It is for this reason why the device or medium supporting propagation of surface modes is collectively known as a slow-wave structure. The examples of slow wave structures include a dielectric material on top of a ground plane and a periodically corrugated structure. Slow wave antennas as well as other devices supporting propagation of an electromagnetic wave with a group velocity equal to the speed of light, i.e., $V_{g}=\mathrm{c}$, are known to be able to generate an endfire radiation [1-7]. This paper focuses on a slow wave endfire antenna having a group velocity $V_{g} \leqslant \mathrm{c}$. Unlike other counterparts including those based on a transition from a microstrip line to spoof SPP waveguide [8-10] and those based on metamaterials [11], the proposed slow wave endfire antenna is to be mounted in the E-plane of a rectangular waveguide.

A variety of spoof SPP-based filters and wave splitters have been realized for SSPP communication systems [12-15]. On the other hand, surface plasmon waveguide based antenna designs, ultra wideband antennas and full-band waveguide differential phase shifters have been proposed recently for microwave applications [16-25]. In [20-25], the maximum bandwidth obtained is around $6-7 \mathrm{GHz}$ with an average gain of $<10 \mathrm{dBi}$.

Open-ended waveguides have been commercially marketed as a directional antenna for terahertz. These components are rarely used in microwave or millimeter wave frequencies in part because of their low gain and limited bandwidth. On the other hands, spoof surface plasmon antennas offer a larger bandwidth with limited gain. As explained in the sections which follow, these two can be combined together to achieve a wide bandwidth, a higher gain and better directional radiation characteristics. Furthermore, the effective aperture is reduced to less than half wavelength in a way to take advantage of the compactness of the design. In this paper, an efficient technique to enhance the bandwidth and gain of an open-ended waveguide using a slow-wave thin plate has been proposed. A simple and efficient endfire radiating hybrid waveguide spoof surface plasmon based design is investigated and analysed. An efficient mode conversion, and transition from waveguide to the proposed spoof surface plasmon design, have been achieved.

\section{Principle}

The Hansen Woodyard condition to achieve endfire radiation gives an optimum phase constant for maximizing the directivity of a lossless continuous endfire array of a particular length. For this, Hansen and Woodyard have proposed that the phase constant be increased from the usual endfire value $\beta=\mathrm{k}$ to an optimum value. For an endfire traveling-wave antenna with a constant phase velocity, there is an optimum velocity ratio $\frac{c}{V_{p h}}$ which results in maximum directivity as shown by Hansen Woodyard for very long antennas and Ehrenspeck-Poehler for an antenna of arbitrary length [1].

Optimum velocity ratio,

$$
c / V_{p h}=1+1 /\left(2 \mathrm{~L} / \lambda_{0}\right)
$$

Using (1), the optimum velocity ratio calculated for the proposed endfire design is

$$
\frac{c}{V_{p h}}=1.1
$$

which satisfies the condition for endfire travelling wave antennas.

The geometry of the proposed design is shown in Figure 1. The design is basically divided into two main parts. First is an open ended rectangular waveguide and the second is a spoof surface plasmon based slow wave thin plate (corrugated antenna). The spoof surface plasmon based corrugated antenna consists of periodically loaded unit cells/elements placed in a regular orientation along the forward $y$-axis. The design is tapered gradually at the end to obtain better reflection characteristic. The transition part of the antenna is also tapered at the beginning of the design which is connected to the open ended rectangular waveguide. This transition part has been optimised to provide better mode conversion from waveguide to corrugated antenna. As shown in Figure 1a, the length of the 
spoof surface plasmon antenna is $130 \mathrm{~mm}$ where the corrugated part is $110 \mathrm{~mm}$ long and the gradually tapered end is $35 \mathrm{~mm}$ long. The front tapered part of the antenna is inserted in the waveguide transition in such a way that it provides better mode conversion and mode matching. The effective aperture of the proposed design is less than half-wavelength i.e., $9 \mathrm{~mm}$. The corrugated design is fabricated over a thin Roger Duroid substrate 5880 with a dielectric constant of 2.2 and thickness $0.1 \mathrm{~mm}$. Figure $1 \mathrm{~b}$ shows the geometry of the waveguide transition designed for the desired operating frequency band.

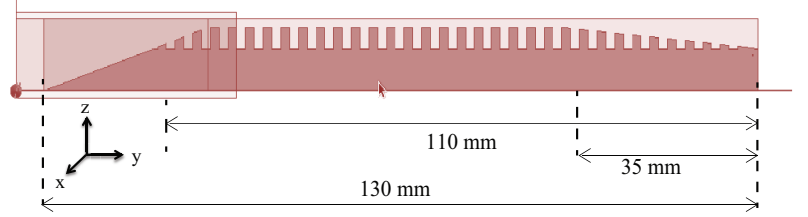

(a)

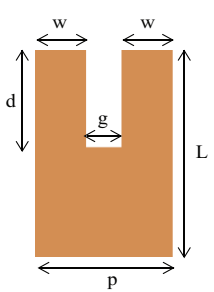

(b)

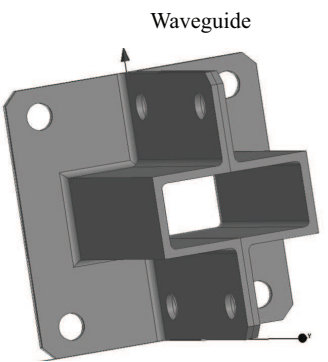

(c)

Figure 1. Geometry of the proposed design (a) Slow wave corrugated design with waveguide transition, (b) Unit element of the spoof surface plasmon (SSP) structure (c) waveguide transition.

Figure 2 shows the dispersion diagram of the periodic unit element of corrugated plate. The unit element has a period ' $p$ ' of $3.2 \mathrm{~mm}$, groove depth 'd' of $3 \mathrm{~mm}$, gap ' $\mathrm{g}$ ' of $1 \mathrm{~mm}$, ' $w$ ' of $1.1 \mathrm{~mm}$ and height ' $\mathrm{L}$ ' of $9 \mathrm{~mm}$. The first two modes are shown in the dispersion graph. The two modes are separated with an airline which defines the slow wave region and fast wave region. To satisfy the endfire condition according to [1], the operating dominant mode should be below the airline and the frequency of operation should be close to the airline. The proposed unit element satisfy the criteria for endfire radiations at frequency $11 \mathrm{GHz}$. Maximum gain and efficiency has been achieved at this frequency.

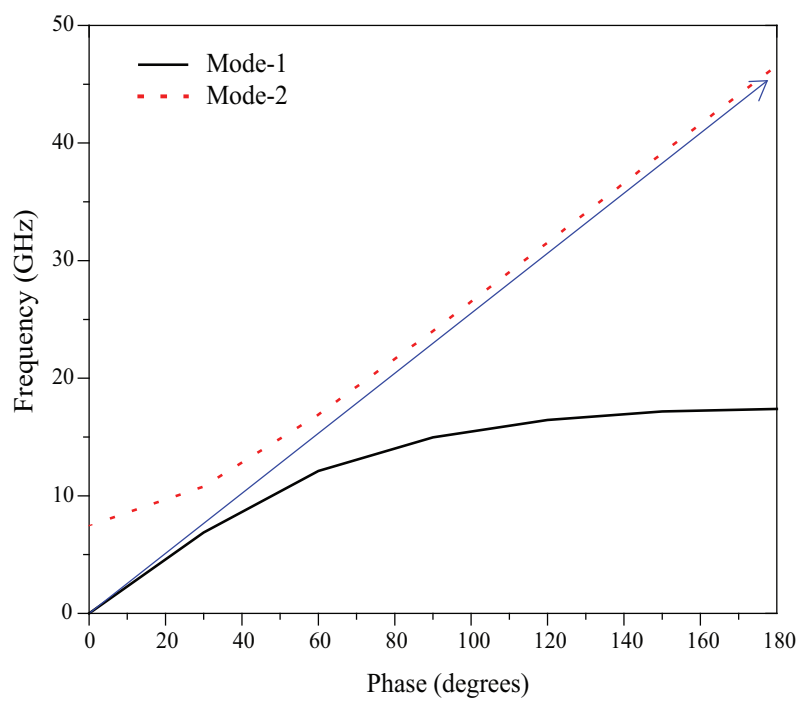

Figure 2. Dispersion curve for the proposed SSP Unit element.

Since the phase velocity remain below the free space velocity, the contributions of the various volume elements of the antenna to the radiation in the forward direction will add constructively 
only up to a certain length of the antenna. For antennas with a gradual taper the optimum length is approximately determined by the condition [2],

$$
\int_{0}^{\mathrm{L}}\left(K_{x}-K_{0}\right)=\pi
$$

where, $K_{x}$ is the phase constant of the guided wave and $K_{o}$ is the free space wave number. If the length is exceeded, destructive interference will occur and a loss of directivity must be expected.

According to the theory, the optimum antenna length of the tapered antennas can be between $3 \lambda$ to $8 \lambda$. Optimum length is approximately $4 \lambda_{0}$ i.e., approx. $130 \mathrm{~mm}$ or less.

Increasing the cross-section of the antenna at the base will enlarge the bandwidth of the antenna and can be used as a method to reduce side lobe levels also (at the expense of a moderate gain reduction).

Furthermore, for long antennas with a gradual taper, it should be of a little consequence if the antenna termination at the far end is blunt or if the antenna is physically continued to its apex. The field strength within this tip section is small since most of its energy is now travelling in the air region.

For short antennas with a length of few wavelengths, tapering to a sharp tip has the advantage of a reduced reflection coefficient.

Here the length of the antenna and the tapering lengths have been optimised to achieve reflection $<-10 \mathrm{~dB}$, broadband and high efficiency ( $>90$ percent). Optimum length has been obtained using the general equation of the tapered antennas which is providing a constructive interference to enhance the gain up to a certain limit.

\section{Parametric Analysis}

This section describes the parametric analysis and analyzes the effect of the length of corrugated plate, effect of transition part, effect of tapering end and peak gain variations with respect to appropriate variables. The simulations have been performed using 3D EM simulation software HFSS (High Frequency Structure Simulator).

Figure 3 shows the variation of S-parameters with the length of the proposed corrugated SSPP antenna. It can be seen from the graph that the length of the antenna is playing a vital role in achieving good reflection parameters. For length $(120 \mathrm{~mm})$, the reflection is not good at all and most of the power is reflected back. If the length is increased $(140 \mathrm{~mm})$, then the reflection is $<-10 \mathrm{~dB}$ but still the antenna is not able to achieve a wideband. At the proposed length of $130 \mathrm{~mm}$, the antenna has achieved a wide bandwidth of about $10 \mathrm{GHz}$.

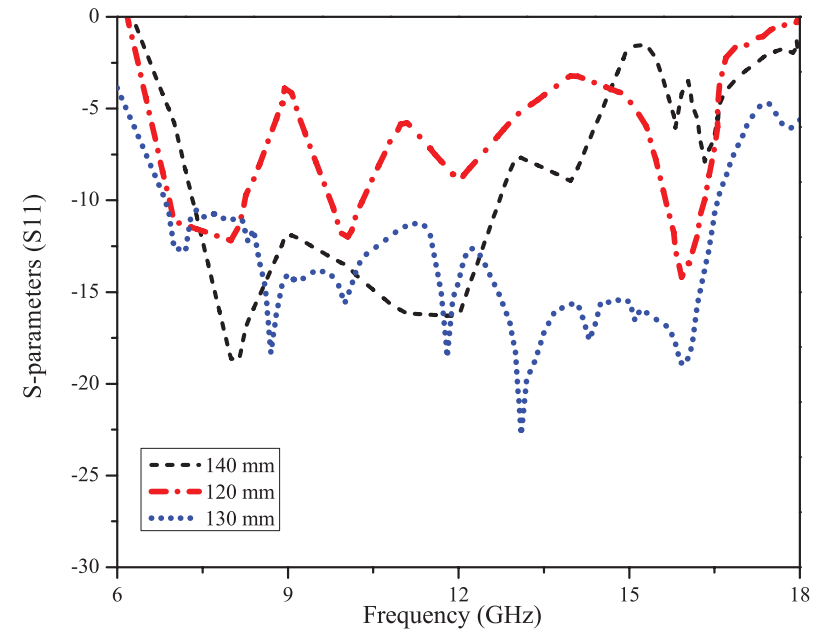

Figure 3. Variation of S-parameters with the length of the corrugated spoof surface plasmon polaritons (SSPP) antenna. 
Figure 4 shows the effect of the tapering in the end part of the finite length antenna. Once the length has been optimized accordingly, the tapering part also needs to be optimized. As can be seen clearly in the graph, the proposed gradual taper $(35 \mathrm{~mm})$ is showing better reflection than a longer taper $(40 \mathrm{~mm})$ although it doesn't have much difference.

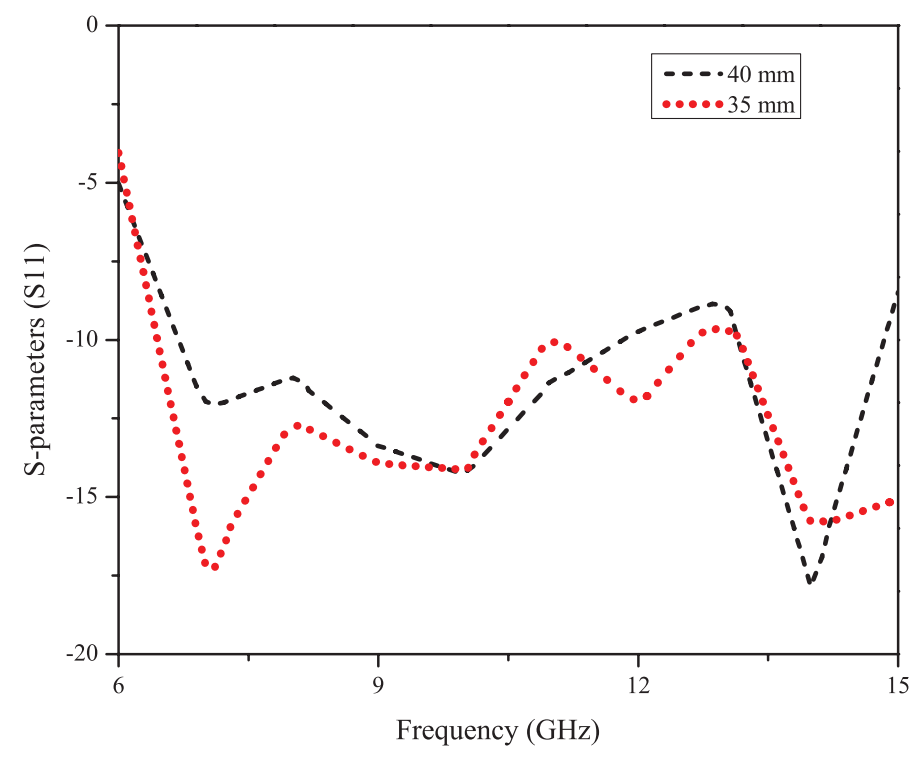

Figure 4. Effect of tapering on reflection parameter.

Figure 5 shows the effect of the transition part at the front of the antenna. Very short transition of $15 \mathrm{~mm}$ has not provided good matching with the waveguide transition whereas a longer transition $30 \mathrm{~mm}$ has achieved better matching. This may be due to the fact that the correct length of tapering results in better mode matching and energy conversion from waveguide transition to the corrugated slow wave antenna design.

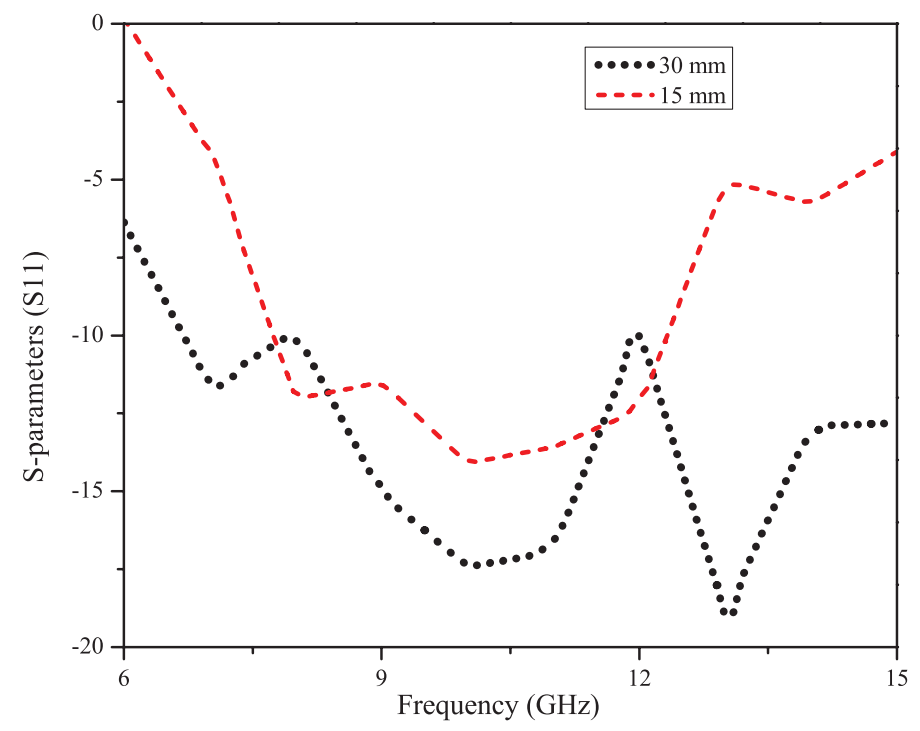

Figure 5. Effect of transition inside waveguide on matching.

Figure 6 shows the gain variations with the length of the proposed design. The peak gain varies a lot with the length of the antenna. This is due to the optimum length, better mode matching and better reflection. The peak gain for the optimum length of $130 \mathrm{~mm}$ is around $14.8 \mathrm{dBi}$ whereas the gain is less than this peak value for other lengths due to the constructive and destructive interference phenomenon. 


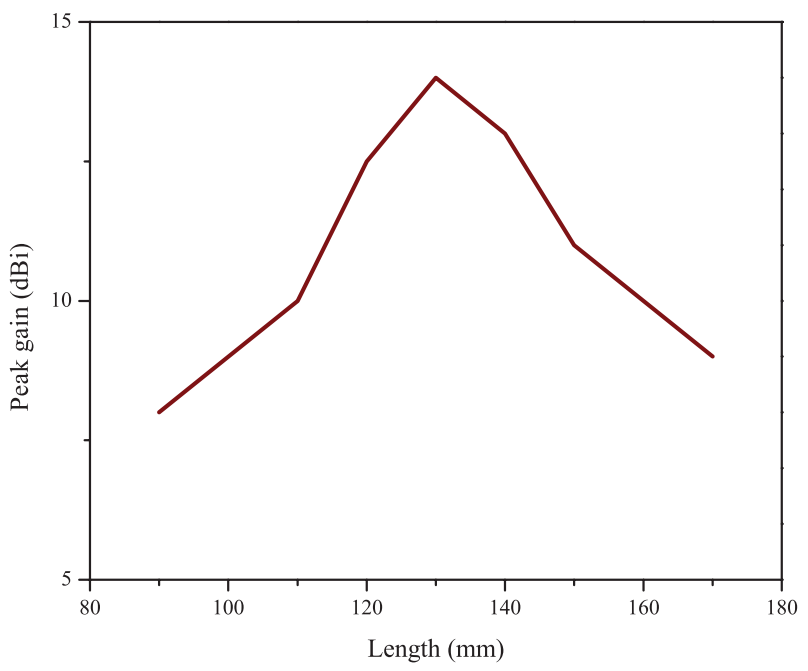

Figure 6. Formulated variation of peak gain with length of SSPP.

Figure 7 shows 2D and 3D-radiation patterns of the simple waveguide and proposed corrugated SSPP at $11 \mathrm{GHz}$ frequency. For comparison purpose, the patterns are shown at $12 \mathrm{GHz}$ also as it is also providing nice endfire radiation with little lower gain. At different frequencies in the operating frequency band, the proposed hybrid waveguide SSP antenna design is showing the endfire or near-endfire radiations. The simulated peak gain of the simple rectangular waveguide is around $7 \mathrm{~dB}$ and for proposed design is $14.8 \mathrm{~dB}$.

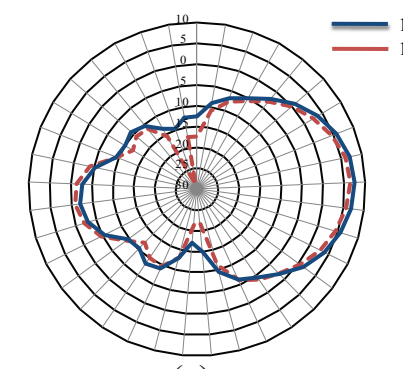

(a)

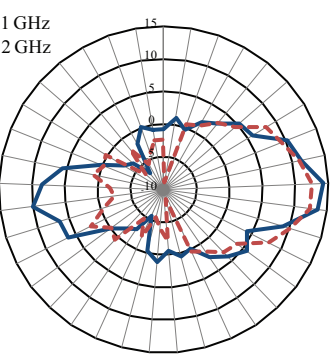

(b)

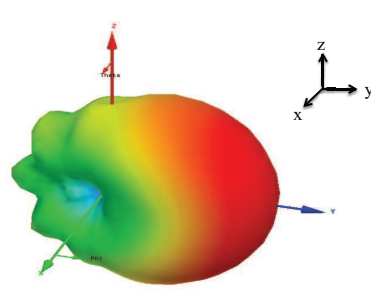

(c)

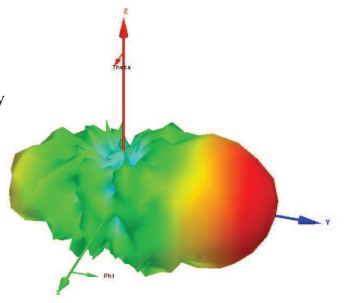

(d)

Figure 7. 2D and 3D Radiation Patterns: (a) Waveguide 2D radiation pattern, (b) Proposed waveguide-SSPP 2D radiation pattern, (c) Waveguide 3D radiation pattern, and (d) Proposed waveguide-SSPP 3D radiation pattern.

Figure 8 shows the E-field distribution for the proposed hybrid waveguide-endfire SSP design along the axis. As can be seen from the plot, the field is highly confined to the corrugated design and has achieved a very good mode matching or energy conversion from the waveguide region to the corrugated surface.

Despite increase in the gain, more back radiation has been observed. Generally, a reflected surface wave spoils the pattern and bandwidth of the antenna. 


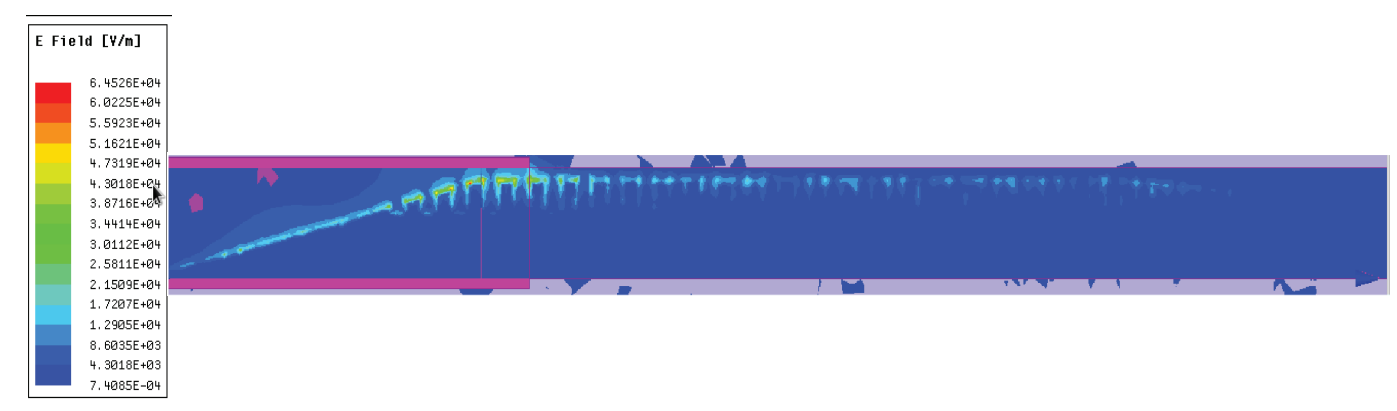

Figure 8. Field distribution: E-Field along the axis.

The optimum formulas derived for the proposed hybrid SSPP design are:

Peak gain $\propto$ Length of the structure (up to a certain limit as criteria)

For normal endfire antennas and broad bandwidth case, directivity (D) in dBi can be written as [2],

$$
\mathrm{D}=10 \log \left(\frac{4 \mathrm{~L}}{\lambda_{0}}\right)
$$

In this design, it can be calculated from (3) as:

$$
\mathrm{D}=10 \log \left(4 \frac{130}{30}\right)=12.4 \mathrm{dBi}
$$

For tapered antennas (or rods), the directivity gain $(\mathrm{G})$ is primarily determined by the antenna length also.

Gain initially increases with L according to (4)

$$
\mathrm{G}=p \frac{\mathrm{L}}{\lambda_{0}} \mathrm{dBi}
$$

From (4), it will be

$$
\mathrm{G}=3.2 \frac{130}{30}=13.9 \mathrm{dBi}
$$

Higher gain has been obtained in the simulation because of gradually tapered end which reduced the reflections to a good extent thereby reducing the back radiations significantly which further leads to gain enhancement.

Similarly half power beamwidth can be calculated as,

$$
\theta=55 \sqrt{\frac{\lambda_{0}}{\mathrm{~L}}}=25 \text { degree }
$$

From the results, it can be seen that the calculated beamwidth is consistent with the measured one and varies from 20 degree to 30 degree.

\section{Experimental Validation}

The design is fully analyzed using a 3D electromagnetic (EM) simulation software HFSS and measured using an Agilent vector network analyzer.

Figure 9 shows the fabricated prototype. Figure $9 a$ is the slow wave corrugated design. Figure $9 b$ is the waveguide transition and Figure $9 \mathrm{c}$ is the combined final design of waveguide and SSPP antenna.

Figure 10 shows the measured S-parameters for the proposed hybrid waveguide-endfire SSP design. From the S-parameters, it is observed that the proposed design has good impedance matching below $-10 \mathrm{~dB}$ in the region from $8 \mathrm{GHz}$ to $18 \mathrm{GHz}$.

Figure 11 shows the experimental setup for the proposed design in the anechoic chamber. 


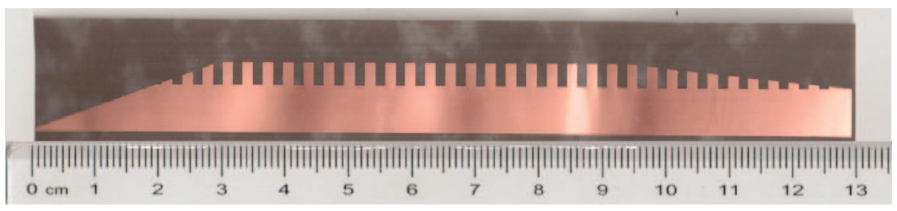

(a)

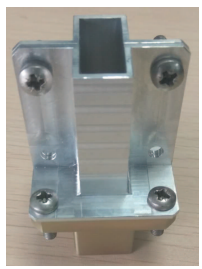

(b)

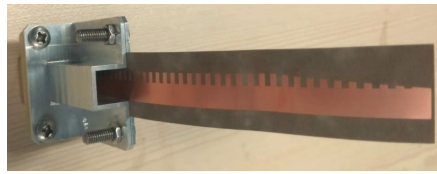

(c)

Figure 9. Prototype of the proposed hybrid waveguide-SSPP (a) corrugated SSPP design, (b) waveguide transition, and (c) proposed final design.

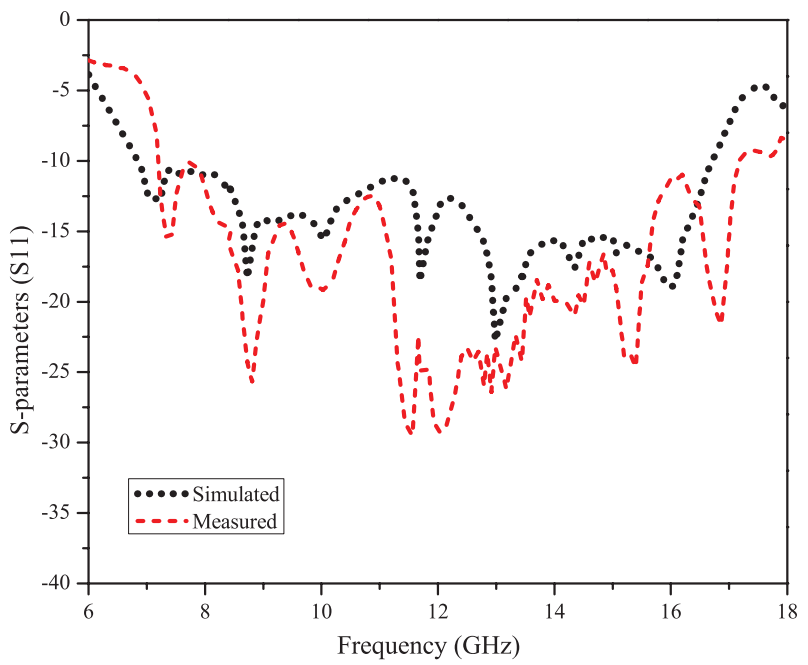

Figure 10. S-parameters of the proposed hybrid waveguide-SSPP design.

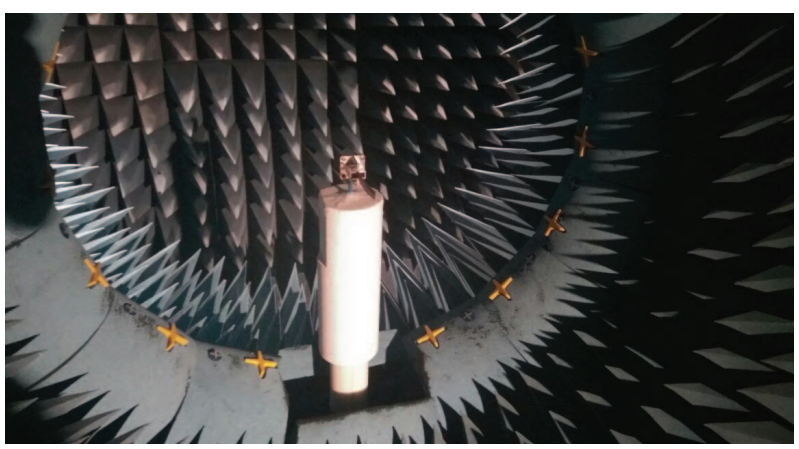

Figure 11. Experimental setup in the anechoic chamber.

Figure 12 shows the radiation patterns (in $\mathrm{dB}$ ) at different frequencies from $8 \mathrm{GHz}$ to $15 \mathrm{GHz}$. It has been observed from the radiation patterns that in the whole operating frequency band, endfire and near endfire radiations have been observed.

Figure 13 shows the comparison of simulated and measured gain for the proposed hybrid waveguide-endfire SSP design. The peak gain for the proposed hybrid design in the operating frequency band obtained is around $14.8 \mathrm{~dB}$. The total gain varies from $7 \mathrm{~dB}$ to $14.8 \mathrm{~dB}$ in operating frequency band. The measured gain is around $13 \mathrm{~dB}$ which is less than simulated one but is close to 
the calculated one. This may be due to the small experimental problems such as connections between waveguide transition and the corrugated design.

Figure 14 shows the efficiency for the proposed hybrid waveguide-endfire SSP design. The radiation efficiency for this design is quite high around 96 percent.

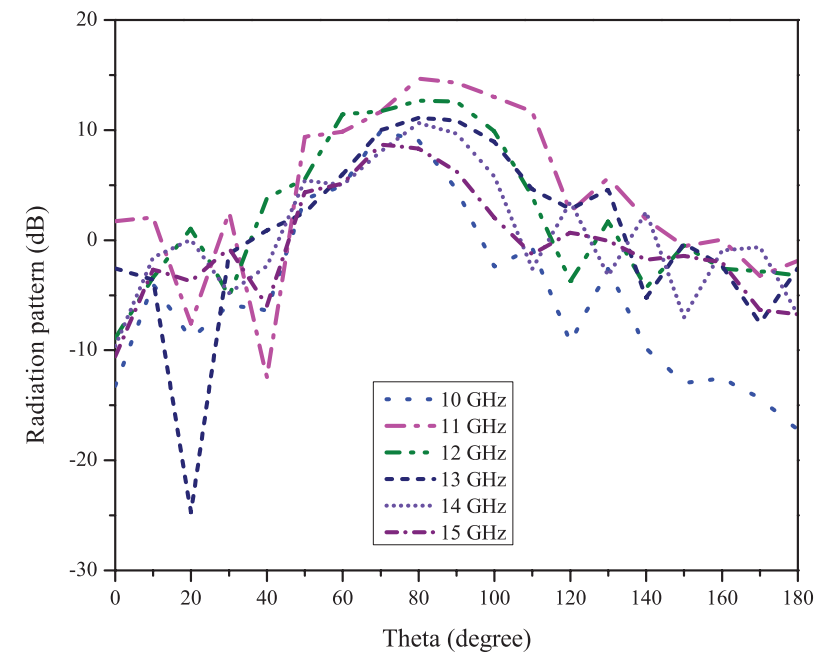

Figure 12. Radiation pattern of the proposed waveguide-SSPP design.

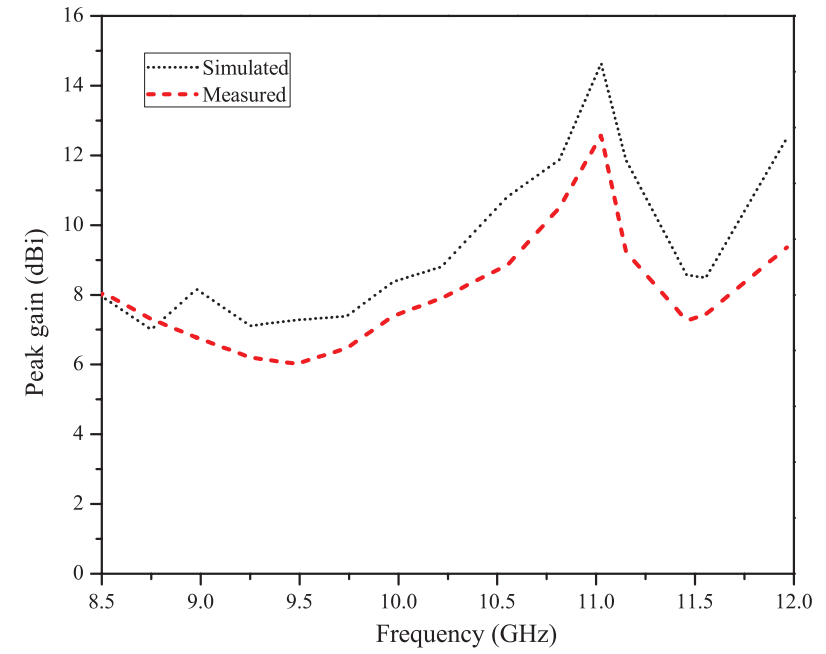

Figure 13. Measured peak gain of the proposed hybrid waveguide-SSPP design.

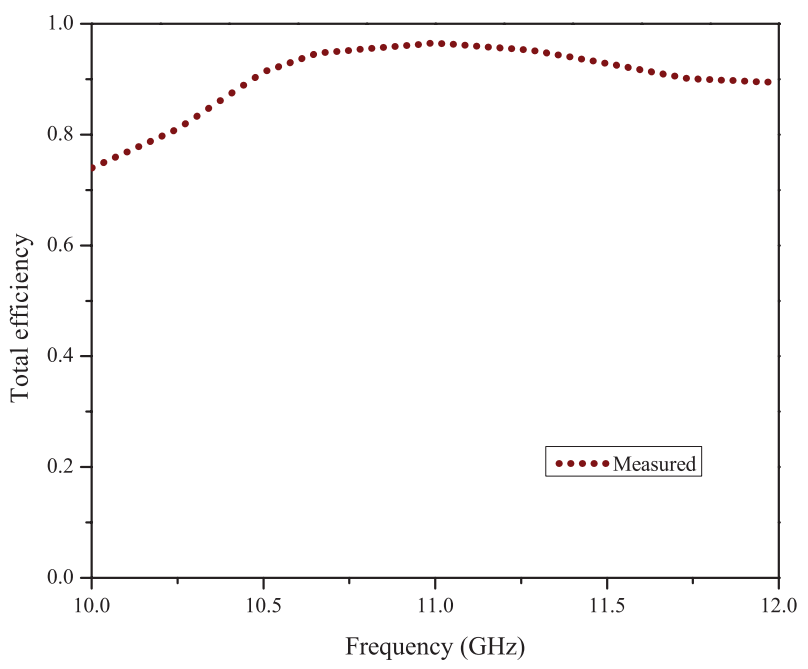

Figure 14. Efficiency of the proposed hybrid waveguide-SSPP design. 


\title{
5. Conclusions
}

A hybrid waveguide design using slow-wave thin corrugated structure has been proposed in this paper. The proposed design has obtained endfire radiations along with an enhanced impedance bandwidth and peak gain. It provides a simple and efficient technique to obtain endfire radiations with improved antenna characteristics using waveguide transition to SSP structure. Peak gain is increased from $7 \mathrm{dBi}$ to $14.8 \mathrm{dBi}$ and a high efficiency of about 96 percent has been obtained.

Author Contributions: A.K. conceived the idea and performed the experiments, Z.N. supervised the work, J.L. and Y.L. made revisions and corrections, L.W.L. helped with simulations and experiments and R.D. performed numerical modeling.

Funding: This work was supported by the National Natural Science Foundation of China under Grant No. 61871375, National Key R\&D Program of China under Grant No. 2018YFC2001002, and Shenzhen Basic Research Project under Grant No. JCYJ20180507182231907.

Conflicts of Interest: The authors declare no conflict of interest.

\author{
Abbreviations \\ SSPP Spoof surface plasmon polaritons \\ SPs Surface Plasmons \\ TLs Transmission lines \\ EM Electromagnetic \\ CPW Coplanar waveguide \\ D Directivity
}

The following abbreviations are used in this manuscript:

\section{References}

1. Hansen, W.W. Radiating Electromagnetic Wave Guide, in Google Patent 2402 622; Wiley: New York, NY, USA, 1946.

2. Zucker, F.J. Surface- and leaky-wave antennas. In Antenna Engineering Handbook; Jasik, H., Ed.; McGraw-Hill: New York, NY, USA, 1961.

3. Kianinejad, A.; Chen, Z.N.; Qiu, C.W. Design and Modeling of Spoof Surface Plasmon Modes-Based Microwave Slow-Wave Transmission Line. IEEE Trans. Microw. Theory Tech. 2015, 63, 1817-1825. [CrossRef]

4. Yin, J.Y.; Ren, J.; Zhang, H.; Pan, B.C. Broadband frequency-selective spoof surface plasmon polaritons on ultrathin metallic structure. Sci. Rep. 2015, 5, 483-486. [CrossRef] [PubMed]

5. Yi, H.; Qu, S.W.; Bai, X. Antenna Array Excited by Spoof Planar Plasmonic Waveguide. IEEE Antennas Wirel. Propag. Lett. 2014, 13, 1227-1230.

6. Cao, W.; Zhang, B.; Liu, A.; Yu, T.; Guo, D.; Wei, Y. Gain Enhancement for Broadband Periodic Endfire Antenna by Using Split-Ring Resonator Structures. IEEE Trans. Antennas Propag. 2012, 60, 3513-3516. [CrossRef]

7. Cao, W. Broadband High-Gain Periodic Endfire Antenna by Using I-Shaped Resonator (ISR) Structures. IEEE Antennas Wirel. Propag. Lett. 2012, 11, 1470-1473.

8. Liao, Z.; Zhao, J.; Pan, B.C.; Shen, X.P.; Cui, T.J. Broadband transition between microstrip line and conformal surface plasmon waveguide. J. Phys. Appl. Phys. 2014, 47, 315103.

9. Zhang, H.C.; Liu, S.; Shen, X.; Chen, L.H.; Li, L.; Cui, T.J. Broadband amplification of spoof surface plasmon polaritons at microwave frequencies. Laser Photonics Rev. 2015, 9, 83-90. [CrossRef]

10. Pan, B.C.; Liao, Z.; Zhao, J.; Cui, T.J. Controlling rejections of spoof surface plasmon polaritons using metamaterial particles. Opt. Express 2014, 22, 13940-13950. [CrossRef]

11. Yin, J.Y.; Bao, D.; Ren, J.; Zhang, H.C.; Pan, B.C.; Fan, Y.; Cui, T.J. Endfire Radiations of Spoof Surface Plasmon Polaritons. IEEE Antennas Wirel. Propag. Lett. 2017, 16, 597-600. [CrossRef]

12. Wu, J.J.; Hou, D.J.; Yang, T.J.; Hsieh, I.J.; Kao, Y.H.; Lin, H.E. Bandpass filter based on low frequency spoof surface plasmon polaritons. Electron. Lett. 2012, 48, 269-270. [CrossRef] 
13. Gao, X.; Zhou, L.; Liao, Z.; Ma, H.F.; Cui, T.J. An ultra-wideband surface plasmonic filter in microwave frequency. Appl. Phys. Lett. 2014, 104, 191603. [CrossRef]

14. Chen, Z.; Hu, Y.; Zheng, X.; Yang, A.; Li, P.; Zhu, L.; Liu, M.; Cheng, L.; Zhou, Q.; Hu, X. Slot-array antenna devising for surface microwave discharge of surface plasmon polaritons. In Proceedings of the 2011 Cross Strait Quad-Regional Radio Science and Wireless Technology Conference, Harbin, China, 26-30 July 2011; Volume 1, pp. 363-366.

15. Wu, J.J.; Hou, D.J.; Chiueh, H.L.; Shen, J.Q.; Wu, C.J.; Kao, Y.H.; Lo, W.C.; Yang, T.J.; Hsieh, I.J. Kind of high directivity scanning radiation based on subwavelength periodic metal structure. Electron. Lett. 2014, 50, 1611-1613. [CrossRef]

16. Xu, J.J.; Zhang, H.C.; Zhang, Q.; Cui, T.J. Efficient conversion of surface-plasmon-like modes to spatial radiated modes. Appl. Phys. Lett. 2015, 106, 021102. [CrossRef]

17. Yin, J.Y.; Zhang, H.C.; Fan, Y.; Cui, T.J. Direct Radiations of Surface Plasmon Polariton Waves by Gradient Groove Depth and Flaring Metal Structure. IEEE Antennas Wirel. Propag. Lett. 2016, 15, 865-868. [CrossRef]

18. Shibayama, J.; Yamauchi, J.; Nakano, H. Metal disc-type splitter with radially placed gratings for terahertz surface waves. Electron. Lett. 2015, 51, 352-353. [CrossRef]

19. Shen, X.; Moreno, G.; Chahadih, A.; Akalin, T.; Cui, T.J. Spoof surface plasmonic devices and circuits in THz frequency. In Proceedings of the 39th International Conference on Infrared, Millimeter, and Terahertz Waves (IRMMW-THz), Tucson, AZ, USA, 14-19 September 2014; p. 1.

20. Guo, Y.J.; Xu, K.D.; Liu, Y.; Tang, X. Novel Surface Plasmon Polariton Waveguides with Enhanced Field Confinement for Microwave-Frequency Ultra-Wideband Bandpass Filters. IEEE Access 2018, 6, 10249-10256. [CrossRef]

21. Zhang, D.; Zhang, K.; Wu, Q.; Ding, X.; Sha, X. High-efficiency surface plasmonic polariton waveguides with enhanced low-frequency performance in microwave frequencies. Opt. Express 2017, 25, 2121-2129. [CrossRef] [PubMed]

22. Gao, X.; Che, W.; Feng, W. Novel non-periodic spoof surface plasmon polaritons with H-shaped cells and its application to high selectivity wideband bandpass filter. Sci. Rep. 2018, 8, 2456. [CrossRef] [PubMed]

23. Cano, J.L.; Mediavilla, A.; Tribak, A. Parametric Design of a Class of Full-Band Waveguide Differential Phase Shifters. Electronics 2019, 8, 346. [CrossRef]

24. Li, K.; Dong, T.; Xia, Z. Wideband Printed Wide-Slot Antenna with Fork-Shaped Stub. Electronics 2019, 8, 347. [CrossRef]

25. Rahman, M.; NagshvarianJahromi, M.; Mirjavadi, S.S.; Hamouda, A.M. Compact UWB Band-Notched Antenna with Integrated Bluetooth for Personal Wireless Communication and UWB Applications. Electronics 2019, 8, 158. [CrossRef]

(C) 2019 by the authors. Licensee MDPI, Basel, Switzerland. This article is an open access article distributed under the terms and conditions of the Creative Commons Attribution (CC BY) license (http://creativecommons.org/licenses/by/4.0/). 